\title{
Burden of illness for patients with cholangiocarcinoma in the United States: a retrospective claims analysis
}

\author{
Christina X. Chamberlain ${ }^{1}$, Elizabeth Faust ${ }^{2}$, Debbie Goldschmidt ${ }^{2}$, Nathan Webster ${ }^{1}$, Audra N. Boscoe ${ }^{1}$, \\ Dendy Macaulay ${ }^{2}$, Mary Linton Peters ${ }^{3}$ \\ ${ }^{1}$ Agios Pharmaceuticals, Inc., Cambridge, MA, USA; ${ }^{2}$ Analysis Group, Inc., New York, NY, USA; ${ }^{3}$ Division of Medical Oncology, Beth Israel \\ Deaconess Medical Center, Boston, MA, USA \\ Contributions: (I) Conception and design: CX Chamberlain, E Faust, D Goldschmidt, D Macaulay, AN Boscoe, ML Peters; (II) Administrative support: \\ E Faust, D Goldschmidt; (III) Provision of study materials or patients: E Faust, D Goldschmidt; (IV) Collection and assembly of data: E Faust, D \\ Goldschmidt; (V) Data analysis and interpretation: All authors; (VI) Manuscript writing: All authors; (VII) Final approval of manuscript: All authors. \\ Correspondence to: Christina X. Chamberlain. Servier Pharmaceuticals, 200 Pier Four Blvd, Boston, MA 02210, USA. \\ Email: christina.chamberlain@servier.com.
}

Background: Advanced cholangiocarcinoma (CCA) is associated with considerable morbidity and mortality. Novel second-line treatments for advanced CCA underscore the need to understand treatment patterns and economic burden of illness in clinical practice.

Methods: This retrospective, claims-based study using Optum's de-identified Clinformatics ${ }^{\circledR}$ Data Mart Database [2007-2019] selected patients with CCA who experienced failure of a line of therapy containing either gemcitabine or fluorouracil. The index date was defined based on evidence of treatment failure: date of last administration of the gemcitabine- or fluorouracil-based regimen plus 28 days, or initiation date of the next-line systemic therapy. Treatment patterns, healthcare resource use (HRU), costs, and survival were assessed during the follow-up period (index until death or end of eligibility).

Results: A total of 1,298 patients met inclusion criteria and had a mean age of 69.1 years. There were 958 patients (73.8\%) with intrahepatic and 275 patients (21.2\%) with extrahepatic CCA. Average follow-up was 7.5 months. Almost $40 \%$ of patients did not receive another line of therapy after the index date. Among the 784 patients who received another line of therapy, $40.3 \%$ used fluorouracil-based therapy, $30.7 \%$ used gemcitabine-based therapy, and $29.3 \%$ used capecitabine-based therapy. Total mean per patient per month CCA-related healthcare costs were $\$ 7,743$, with medical services $(\$ 6,685)$ a larger driver of monthly costs relative to treatment costs $(\$ 1,058)$. Median overall survival (OS) was 5.3 months among all patients.

Conclusions: Many patients with advanced CCA do not initiate additional therapy after failure of gemcitabine or fluorouracil treatment, and there is considerable variation in treatments among those who do. This study highlights the high costs and unmet need for a standard of care in this patient population.

Keywords: Cholangiocarcinoma (CCA); economic burden; treatment patterns; burden of illness

Submitted Nov 24, 2020. Accepted for publication Jan 18, 2021.

doi: 10.21037/jgo-20-544

View this article at: http://dx.doi.org/10.21037/jgo-20-544

\section{Introduction}

Cholangiocarcinoma (CCA) is a rare cancer that originates in the bile ducts (1). It is typically classified based on anatomical location as intrahepatic CCA (ICC) or extrahepatic CCA (ECC) $(2,3)$. The overall incidence rate of CCA is low [approximately $1-2.4$ per 100,000 in the United States (US)], but has increased over the past four decades (4-8). Due to errors in classification and difficulty in diagnosis, the actual incidence rate may be higher (8).

CCA is asymptomatic in its early stages, typically diagnosed in older adults ( $>65$ years) and is usually 
advanced at the time of diagnosis $(4,9,10)$. The prognosis for CCA is poor, with 5-year overall survival (OS) rates of approximately $10 \%$ (11). Currently, there are few treatment options available for CCA, mainly limited to chemotherapybased regimens, though a number of targeted therapies have recently been developed $(12,13)$. Surgical resection is the only potentially curative therapy, but it is feasible in only approximately one-third of patients, and rates of recurrence are high $(14,15)$.

For patients with unresectable or advanced CCA, chemotherapy (locoregional or systemic) is the main treatment option (14). In particular, gemcitabine plus cisplatin is recommended as the primary treatment per the NCCN Clinical Practice Guidelines in Oncology (NCCN Guidelines $^{\circledR}$ ) for Hepatobiliary Cancers (15-17). However, there is no clear standard of care for subsequent lines, and current chemotherapy-based second-line options, including a combination of modified (i.e., varying) doses of leucovorin calcium, fluorouracil, and oxaliplatin (mFOLFOX), are associated with limited survival benefit (18). The NCCN Guidelines ${ }^{\circledR}$ include newer targeted treatments for subsequent lines, which may be useful for patients with specific mutations (17). Specifically, pemigatinib was recently approved in the US for previously-treated patients with fibroblast growth factor receptor 2 (FGFR2) rearrangement/fusion CCA, and ivosidenib is being investigated in a Phase III clinical trial (ClarIDHy) for previously-treated patients with isocitrate dehydrogenase 1 mutation (mIDH1) CCA $(12,13,19)$.

In addition to the unmet treatment needs of patients with CCA, the disease is associated with a considerable economic burden that has increased over time. One US study found that the mean number of CCA-related hospital admissions increased by $16 \%$ and mean CCA-related inflation-adjusted hospital charges increased by $113 \%$ from 1997 to 2012 (20). This aligns with another study examining trends in CCArelated hospitalizations in the US (2000 to 2014) that found the number of hospitalizations related to ICC increased by $86 \%$, and charges for both ICC and ECC increased significantly over time (21). However, there are significant gaps in the literature describing the healthcare resource use (HRU) and costs associated with CCA as related studies are dated, do not focus specifically on advanced CCA, and only assess CCA-related hospitalizations, not other sites of care or treatment patterns. This study addresses the gaps and describes treatment patterns, HRU in all settings, and costs associated with previously-treated advanced CCA among adults in the US. Survival for this population was also evaluated.

We present the following article in accordance with the Strengthening the Reporting of Observational Studies in Epidemiology (STROBE) reporting checklist available at: http://dx.doi.org/10.21037/jgo-20-544.

\section{Methods}

This retrospective study used data from Optum's deidentified Clinformatics ${ }^{\circledR}$ Data Mart Database (Q12007Q12019), a commercial and Medicare Advantage insurance claims database consisting of approximately 15 million beneficiaries in any given year. The covered population is located predominantly in the South and North Central (Midwest) US census regions, although there are beneficiaries from all fifty states. Complete medical and pharmacy claims are available for all members, including those 65 years of age or older with Medicare Advantage. The database also contains linked information related to mortality. Cost data are based on standardized payments.

Adult patients with CCA who were previously treated with and had failure of a line of therapy containing either gemcitabine or fluorouracil (5-FU), suggesting possible advanced disease, were included. Specifically, patients with CCA were identified based on having at least 3 claims with an International Classification of Diseases, 9/10th Revision (ICD-9/10) diagnosis code of ICC (ICD-9 155.1, ICD-10 C22.1), ECC (ICD-9 156.1, ICD-10 C24.0), or undefined CCA (ICD-10 C24.8, C24.9) on distinct days within one year of the first CCA diagnosis observed in the data.

Patients were required to have undergone treatment with a systemic regimen containing gemcitabine or 5 -FU following diagnosis for CCA (defined here as first-line systemic therapy), and then to have either received another line of systemic chemotherapy treatment or died during follow-up (suggesting treatment failure). The next line of systemic therapy was defined as the initiation of at least one new chemotherapeutic agent not present in the first-line therapy. For combination regimens, discontinuation of one of the agents was not considered a new line of therapy. The index date was defined as the last administration of the firstline regimen plus 28 days, or the initiation of the next-line systemic therapy, whichever occurred first. For patients who had a surgical resection, including hepatic wedge resection, Whipple procedure, or bile duct resection, adjuvant chemotherapy was not considered first-line systemic 
therapy unless a next line was started less than six months after completion of the adjuvant regimen, suggesting rapid disease progression. In this scenario, the index date was defined as the initiation date of this next-line therapy.

Patients were also required to have had at least 9 months of continuous health plan enrollment, with at least 6 months prior to and at least 3 months following the index date. Patients who died within 3 months of the index date were also included in the study sample as they were considered to have failure of first-line therapy. The 6 months prior to the index date were defined as the baseline period. The time from the index date until death or end of health plan enrollment/eligibility was defined as the follow-up period (Figure S1).

To ensure the sample was limited to patients with failure of gemcitabine- or 5-FU-based therapy, patients were excluded if they were alive at the end of the followup period with no evidence of initiating a next line of therapy. In addition, to avoid including patients with potential misdiagnosis, patients with an ECC diagnosis code and a procedure code for cholecystectomy or distal pancreatectomy, but no evidence of a resectable ECC surgery (e.g., Whipple procedure, bile duct resection), were excluded.

Patient demographics, clinical characteristics and CCArelated treatment use during the baseline period were assessed. Treatment patterns during the follow-up period included the number of lines of systemic therapy on or after the index date. For the first two lines of therapy on or after the index date, the duration of each line and proportion of patients receiving each type of regimen were described. For those patients for whom the index date was defined as the last administration of first-line regimen plus 28 days, the time from index date to second-line therapy was described.

For HRU, the percentage of patients with each type of medical visit [e.g., inpatient admissions and subset of intensive care unit (ICU) admissions, emergency room visits, outpatient visits, home health/hospice, radiographic services] during the follow-up period was summarized. Monthly HRU [e.g., average number per patient per month $(\mathrm{PPPM})]$ was also assessed by calculating the total number of each type of admission or visit divided by months of follow-up for each patient. Medical visits were defined as CCA-related if any claim associated with that visit had a diagnosis code for CCA. Both all-cause and CCA-related HRU were reported.

Monthly all-cause and CCA-related healthcare costs included medical service costs (e.g., inpatient admissions and subset of ICU admissions, emergency room visits, outpatient visits, home health/hospice, radiographic services, and other medical services) and treatment costs. Costs were separately summarized by patient age $(<65$, $\geq 65$ years) and site of disease (ICC, ECC). Costs were adjusted for inflation (2019 USD) using the US Medical Care consumer price index from the Bureau of Labor Statistics from the US Department of Labor (22). Lastly, patient survival was measured from the index date to death, with patients censored at the end of data coverage.

\section{Statistical analysis}

Continuous variables were described using means, medians, and standard deviations (SD); categorical variables were described using frequencies and percentages. Kaplan-Meier $(\mathrm{KM})$ analyses were used to evaluate survival.

\section{Ethical statement}

The study was conducted in accordance with the Declaration of Helsinki (as revised in 2013). The deidentified data did not require approval from an ethics board.

\section{Results}

A total of 1,298 patients met the inclusion criteria, 108 of whom $(8.3 \%)$ had resection prior to initiating a line of gemcitabine- or 5-FU-based therapy. Mean follow-up was 229 days (median 140 days). There were 958 patients (73.8\%) with ICC, 275 patients $(21.2 \%)$ with ECC, and 65 patients $(5.0 \%)$ with undefined CCA. Patients had a mean age of 69.1 years, with the majority of patients $(72.3 \%) \geq 65$ years, and $51 \%$ female. Common CCA-related comorbidities included hypertension (70.3\%), liver disease $(60.7 \%)$, and coronary heart disease $(24.9 \%)$. A total of 1,080 patients $(83.2 \%)$ were treated with a gemcitabinebased regimen and $224(17.3 \%)$ were treated with a $5-\mathrm{FU}-$ based regimen in the first line (6 patients received both treatments) (Table 1). There were $84.8 \%$ of patients with ICC and $78.2 \%$ of patients with ECC who received a gemcitabine-based first-line regimen. Similarly, there were $85.2 \%$ of patients $\geq 65$ and $78.0 \%$ of patients $<65$ years old who received a gemcitabine-based first-line regimen.

A total of 514 patients (39.6\%) died during the followup period without receiving a second-line therapy. Among the remaining sample (784 patients), 510 patients (65.1\%) 
Table 1 Baseline ${ }^{\dagger}$ patient characteristics and treatment use

\begin{tabular}{|c|c|}
\hline Characteristics & $\begin{array}{c}\text { All patients } \\
(\mathrm{N}=1,298)\end{array}$ \\
\hline Age at index date, mean \pm SD & $69.1 \pm 10.0$ \\
\hline \multicolumn{2}{|l|}{ Gender, N (\%) } \\
\hline Female & $661(50.9)$ \\
\hline Male & $636(49.0)$ \\
\hline Unknown & $1(0.1)$ \\
\hline \multicolumn{2}{|l|}{ Type of $C C A^{\ddagger}$} \\
\hline Intrahepatic & $958(73.8)$ \\
\hline Extrahepatic & $275(21.2)$ \\
\hline Undefined & $65(5.0)$ \\
\hline \multicolumn{2}{|l|}{ Year of index date, $\mathrm{N}(\%)$} \\
\hline $2007-2010$ & $244(18.8)$ \\
\hline 2011-2014 & $411(31.7)$ \\
\hline 2015-2019 & $643(49.5)$ \\
\hline \multicolumn{2}{|l|}{ Insurance type $e^{\S}, \mathrm{N}(\%)$} \\
\hline Commercial & $362(27.9)$ \\
\hline Medicare & $940(72.4)$ \\
\hline \multicolumn{2}{|l|}{ Geographic region ${ }^{\S}, \mathrm{N}(\%)$} \\
\hline Northeast & $183(14.1)$ \\
\hline Midwest & $371(28.6)$ \\
\hline South & $486(37.4)$ \\
\hline West & $255(19.6)$ \\
\hline Unknown & $9(0.7)$ \\
\hline \multicolumn{2}{|c|}{ Common CCA-related comorbidities, N (\%) } \\
\hline Hypertension & $912(70.3)$ \\
\hline Liver disease & $788(60.7)$ \\
\hline Coronary heart disease & $323(24.9)$ \\
\hline Radiotherapy, N (\%) & $227(17.5)$ \\
\hline \multicolumn{2}{|l|}{ Surgery, N (\%) } \\
\hline Whipple procedure & $30(2.3)$ \\
\hline Hepatic wedge resection & $36(2.8)$ \\
\hline Bile duct resection & $4(0.3)$ \\
\hline
\end{tabular}

Table 1 (continued)
Table 1 (continued)

\begin{tabular}{|c|c|}
\hline Characteristics & $\begin{array}{c}\text { All patients } \\
(\mathrm{N}=1,298)\end{array}$ \\
\hline \multicolumn{2}{|c|}{ First-line systemic therapy ${ }^{\S \uparrow}$ including, N (\%) } \\
\hline Gemcitabine & 1,080 (83.2) \\
\hline Fluorouracil & 224 (17.3) \\
\hline \multicolumn{2}{|c|}{$\begin{array}{l}{ }^{\dagger} \text {, the baseline period is the } 6 \text { months prior to index date; }{ }^{\ddagger} \text {, } \\
\text { for patients with diagnosis codes for both ICC and ECC, their } \\
\text { classification as having ICC or ECC was based on the most } \\
\text { recent code prior to the index date; }{ }^{\S} \text {, patients may have more } \\
\text { than one insurance type and more than one geographic region } \\
\text { during the baseline period. Additionally, patients may be } \\
\text { categorized under both gemcitabine-based and fluorouracil- } \\
\text { based therapy if the combination included both treatments. } \\
\text { ", first-line systemic therapy is defined in this study as the } \\
\text { line of therapy containing either gemcitabine or fluorouracil } \\
\text { that resulted in treatment failure as of the index date. CCA, } \\
\text { cholangiocarcinoma; ECC, extrahepatic cholangiocarcinoma; } \\
\text { ICC, intrahepatic cholangiocarcinoma; SD, standard deviation. }\end{array}$} \\
\hline
\end{tabular}

received one subsequent line of therapy during the followup period, and 274 patients (34.9\%) received two or more subsequent lines of therapy. Among the 784 patients who received second-line therapy, regimens including infusional 5-FU were used by the largest proportion of patients $(40.3 \%)$, while regimens including gemcitabine or capecitabine were each used by approximately $30 \%$ of patients. Patients were treated with the second-line therapy for a median of 56 days. Among the 274 patients with a third line of therapy, regimens including gemcitabine or 5 -FU were each used by approximately $38 \%$ of patients. Median treatment duration was similar to second-line (49 days) (Table 2).

There were $502(38.7 \%)$ patients with a CCA-related inpatient admission and 778 patients $(59.9 \%)$ with a CCArelated outpatient visit during the follow-up period. Patients had an average of 0.2 CCA-related inpatient admissions and 1.0 CCA-related outpatient visit per month. Among the patients with a CCA-related inpatient admission, the average length of stay per admission was 4.4 days, and over one-third of these patients spent time in the ICU (Table 3). The total mean all-cause PPPM healthcare costs were $\$ 14,403$. CCA-related PPPM costs $(\$ 7,743)$ were over half of the total all-cause costs, with medical services (mean 
Table 2 Treatment patterns during follow-up period

\begin{tabular}{|c|c|}
\hline & All patients $(\mathrm{N}=1,298)$ \\
\hline \multicolumn{2}{|l|}{ Systemic therapy during the follow-up period, $N(\%)$} \\
\hline Number of lines of therapy identified on or after the index date, mean \pm SD [median] & $0.9 \pm 1.1[1.0]$ \\
\hline 0 lines of therapy identified on or after index date, $\mathrm{N}(\%)$ & $514(39.6)$ \\
\hline$\geq 2$ lines of therapy identified on or after index date, $N(\%)$ & $274(21.1)$ \\
\hline \multicolumn{2}{|l|}{ Second-line systemic therapy ${ }^{\dagger}$} \\
\hline Patients receiving second-line therapy, N (\%) & $784(60.4)$ \\
\hline \multicolumn{2}{|l|}{ Second-line systemic therapy ${ }^{\dagger}$ including, $N(\%)$} \\
\hline Capecitabine & $230(29.3)$ \\
\hline Other systemic therapy ${ }^{\ddagger}$ & $52(6.6)$ \\
\hline Duration of second-line systemic therapy ${ }^{\dagger}$ (days), mean \pm SD [median] & $87.9 \pm 104.4[56.0]$ \\
\hline $\begin{array}{l}\text { Time from index date to second-line therapy among patients with second-line initiation after index date } \\
\text { (days), mean } \pm \text { SD [median] }\end{array}$ & $92.6 \pm 142.6[35.0]$ \\
\hline \multicolumn{2}{|l|}{ Third-line systemic therapy", N (\%) } \\
\hline Patients receiving $\geq 2$ lines of therapy on or after the index date & $274(21.1)$ \\
\hline \multicolumn{2}{|l|}{ Third-line systemic therapy" including, N (\%) } \\
\hline
\end{tabular}

${ }^{\dagger}$, second-line systemic therapy is defined in this study as the next-line of treatment after failure of the first-line systemic therapy containing either gemcitabine or fluorouracil. Categories are not mutually exclusive; ${ }^{\ddagger}$, other systemic therapy regimens including irinotecan, nabpaclitaxel, and/or oxaliplatin; ${ }^{\S}, 274$ patients initiated a second-line therapy after the index date; 510 patients had index date defined as initiation of second-line therapy. ", third-line systemic therapy is defined in this study as the next-line treatment after second-line therapy defined above. Categories are not mutually exclusive. SD, standard deviation.

$\$ 6,685)$ being a larger driver of monthly CCA-related costs relative to treatment costs (mean $\$ 1,058$ ) (Table 4). The main medical service monthly cost drivers were inpatient (mean \$3,529) and outpatient costs (mean \$2,212). CCArelated healthcare costs were higher for patients $<65 v s$. $\geq 65$ years, driven by both CCA-related resource use and systematic treatment costs (Figure 1, Tables S1 and S2). There were no substantial differences in healthcare costs observed between patients with ICC and ECC.
Three-quarters of patients $(74.5 \%)$ died during the follow-up period. The median OS was 5.3 months [95\% confidence interval (CI): 4.8-5.8 months], with 6- and 12 -month OS rates of $46.7 \%$ and $28.9 \%$, respectively. Among the 784 patients who received a second line of therapy, $57.8 \%$ died during the follow-up period and median OS was 10.2 months (95\% CI: 9.4-12.0 months). Median OS was 1.8 months (95\% CI: 1.6-2.1 months) for the 514 patients who did not receive a second-line therapy 
Table 3 HRU during the follow-up period

\begin{tabular}{|c|c|c|}
\hline & CCA-related $^{\dagger}(\mathrm{N}=1,298)$ & All-cause $(\mathrm{N}=1,298)$ \\
\hline Inpatient admission & $502(38.7)$ & $830(63.9)$ \\
\hline ICU admission & $186(14.3)$ & $265(20.4)$ \\
\hline Emergency room visit & $143(11.0)$ & $512(39.4)$ \\
\hline Outpatient visit & 778 (59.9) & $1,096(84.4)$ \\
\hline Radiology visit & $573(44.1)$ & $937(72.2)$ \\
\hline \multicolumn{3}{|c|}{ Number of medical services per patient per month ${ }^{\S}$, mean $\pm S D$} \\
\hline Inpatient admissions & $0.2 \pm 1.1$ & $0.5 \pm 2.0$ \\
\hline Emergency room visits & $0.0 \pm 0.1$ & $0.1 \pm 0.3$ \\
\hline Home health care/hospice visits & $0.1 \pm 0.4$ & $0.2 \pm 1.1$ \\
\hline Outpatient visits & $1.0 \pm 1.9$ & $2.3 \pm 2.5$ \\
\hline Radiology visits & $0.2 \pm 0.4$ & $0.6 \pm 1.1$ \\
\hline
\end{tabular}

${ }^{\dagger}$, if any claim within an admission/visit had a diagnosis code for CCA, the entire visit was considered CCA-related; ${ }^{\ddagger}$, patients with any of the following medical services during their follow-up period were summarized; patients have varying length of follow up; ${ }^{\S}$, per patient per month HRU was calculated based on the average of the number of admission(s) or visit(s) per patient divided by number of months in each patient's follow-up; ", length of stay for inpatient and ICU admissions were calculated only among patients with at least one inpatient or ICU admission, respectively. CCA, cholangiocarcinoma; HRU, healthcare resource utilization; ICU, intensive care unit; SD, standard deviation.

(all of whom died during the follow-up period).

\section{Discussion}

This retrospective claims-based study characterizes current treatment patterns, HRU, healthcare costs, and OS among patients with previously-treated advanced CCA in the US. Following failure of an initial gemcitabine- or 5-FU-based regimen, chemotherapy regimens varied with gemcitabine, capecitabine, or infusional 5-FU being commonly used in subsequent lines; however, there was a substantial percentage of patients (nearly $40 \%$ ) who did not receive a second-line therapy. The large percentage of patients without second-line therapy coupled with the high CCArelated medical services costs and short OS underscore the need for more effective treatment options in this patient population.

This is the first study to present all-cause and CCA- related HRU and costs for this patient population, so direct comparisons cannot be made to existing studies; however, these findings align with prior studies highlighting the high economic burden associated with CCA $(20,21,23)$. All-cause costs are nearly double CCA-related costs, which may be explained in part by underlying health issues associated with CCA (e.g., hypertension and liver disease) that contribute to the overall burden as well as common complications of disease including cholangitis, biliary obstruction, and abdominal pain. Patients with CCA had high allcause inpatient utilization, and the majority of their ICU admissions were attributed to CCA. The biggest driver of CCA-related healthcare costs was medical services relative to treatment costs. Our study also found patients younger than 65 had higher CCA-related costs than patients 65 and older, possibly due to the management of younger patients with more intensive (and expensive) treatments which may not be appropriate for older patients, as well as the 
Table 4 Monthly healthcare costs per patient during the follow-up period

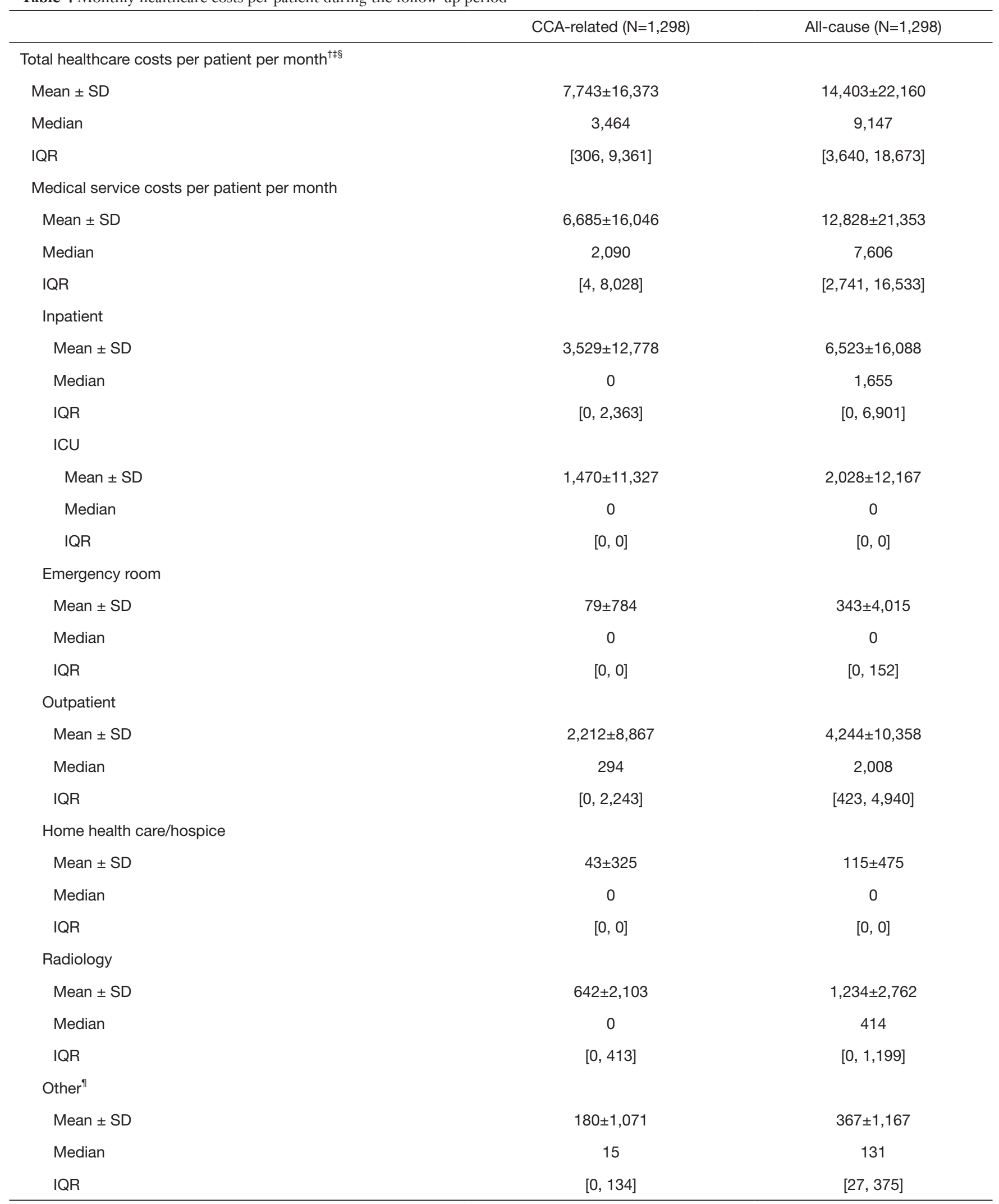

Table 4 (continued)

(C) Journal of Gastrointestinal Oncology. All rights reserved. 
Table 4 (continued)

\begin{tabular}{lcr}
\hline & CCA-related $(\mathrm{N}=1,298)$ & All-cause $(\mathrm{N}=1,298)$ \\
\hline Treatment costs per patient per month & & \\
Mean \pm SD & $1,058 \pm 2,912$ & $1,575 \pm 3,199$ \\
Median & 172 & 584 \\
IQR & {$[0,1,279]$} & {$[64,2,022]$} \\
\hline
\end{tabular}

${ }^{\dagger}$, if any claim within a visit had a diagnosis code for CCA, the entire visit was considered CCA-related; ${ }^{\ddagger}$, all costs were inflated to 2019 USD; ${ }^{\S}$, per patient per month costs were calculated based on the average of the costs per patient for each admission or visit type divided by number of months in each patient's follow-up; ", "Other" refers to the following types of services: durable medical equipment, services and supplies, transportation services, laboratory services, and dental services; ", CCA-related treatment costs included systemic treatments only. CCA, cholangiocarcinoma; ICU, intensive care unit; SD, standard deviation; USD, United States dollars.

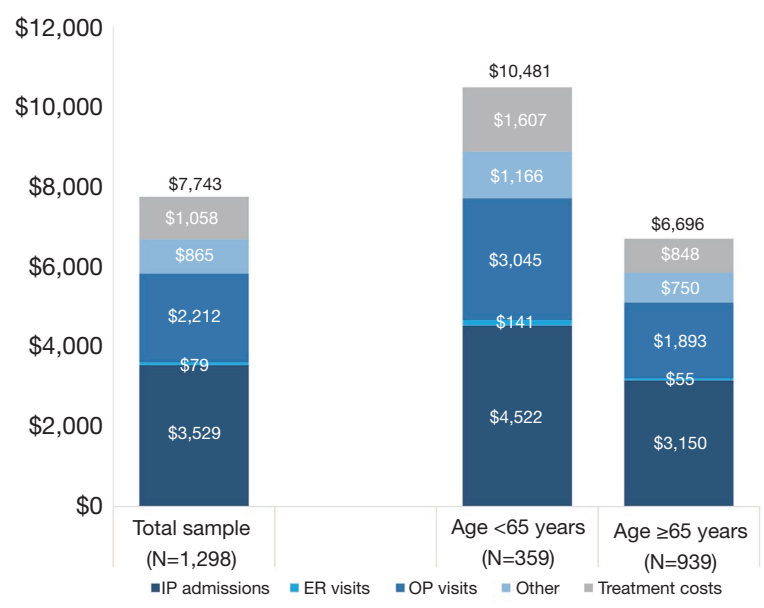

Figure 1 Mean monthly CCA-related healthcare costs ${ }^{\dagger \ddagger}$ per patient during the follow-up period stratified by age. ${ }^{\dagger}$, if any claim within a visit had a diagnosis code for CCA, the entire visit was considered CCA-related; ${ }^{\ddagger}$, all costs were inflated to 2019 USD. CCA, cholangiocarcinoma; ER, emergency room; IP, inpatient; OP, outpatient; USD, United States dollars.

management of symptoms and side effects associated with these regimens.

Although chemotherapy, particularly with gemcitabine plus cisplatin, is considered standard of care for frontline treatment of patients with advanced or metastatic CCA, there is no consensus regarding specific treatment combinations/sequences in later lines $(18,24)$. The variation in second-line treatments reported in this study aligns with a retrospective chart review study of patients receiving second-line treatment for advanced CCA (25). All patients in that study received a second-line therapy by design, but the variation was evident: $19.6 \%$ received gemcitabine plus platinum, $28.6 \%$ received gemcitabine plus fluoropyrimidine, $37.5 \%$ received another fluoropyrimidine combination regimen, and $14.3 \%$ of patients received other treatment combinations (25). For the majority of patients with advanced or metastatic CCA, there are limited treatment options beyond intravenously infused chemotherapy, which typically provides only modest survival benefits and substantial toxicity, contributing to the burden of illness in this patient population. New and upcoming targeted treatments may provide improvements for at least some patients. Pemigatinib was approved recently for treatment of CCA with FGFR2 rearrangement/ fusion, a mutation that occurs almost exclusively in ICC and is observed in $10-16 \%$ of these patients $(13,26-28)$. Ivosidenib is under investigation for CCA with mIDH1, a mutation that is prevalent in approximately $18 \%$ of patients with ICC and $1 \%$ of patients with ECC in the US (29). These oral, targeted therapies may be cost-effective options for improving disease control, as has been seen in other tumor types (e.g., non-small-cell lung carcinoma) (30).

The poor OS reported here is consistent with other studies $(18,31)$ that have assessed survival in advanced or metastatic CCA despite differences in methodological design. In this study, OS was assessed among a population of patients starting from the time of assumed first-line therapy failure, not necessarily from the start of subsequentline treatment initiation commonly used in clinical trials; nonetheless, the median OS of 5.3 months is poor. A phase III clinical trial comparing mFOLFOX to supportive care as second-line therapy for patients with biliary tract cancer estimated median OS of 6.2 and 5.3 months, respectively (18). A phase II clinical trial assessed OS with capecitabine + irinotecan + gemcitabine + bevacizumab in previouslytreated patients and estimated median OS of 7.7 months in 
patients with CCA (31). Collectively, these studies suggest that many currently available second-line chemotherapy treatment options have limited survival benefits. A substantial proportion of our sample did not initiate a second-line treatment and had shorter OS (1.8 months), further supporting the need for additional second-line treatment options. Emerging targeted treatments, such as oral pemigatinib and oral ivosidenib, have the potential to improve survival among certain CCA populations $(12,13)$.

This study should be considered within the context of limitations that are inherent to retrospective observational claims-based studies. Additional considerations specific to this study design should also be noted. First, retrospective databases are subject to coding errors or data omissions. Relatedly, visits were defined as CCA-related if any claim within that visit had a diagnosis code for CCA; therefore, whether a visit was CCA-related was determined by physician coding practices. Second, healthcare resources and treatments received in clinical trials were not captured in the data. It is unknown what percentage of the study population may have been enrolled in a clinical trial. Third, the sample selection algorithm was designed to identify patients with $\mathrm{CCA}$ and failure of treatment with gemcitabine- or 5-FUbased therapies (i.e., indication of possible advanced disease) by using treatment discontinuation, treatment switch, or death as a proxy for failure. However, this algorithm may not have identified all patients of interest, and therefore results may not be fully representative of the intended population. Lastly, the focus of the study was advanced CCA requiring systemic treatment after an initial line of therapy, and patients who were alive at the end of the follow-up period with no evidence of initiating a new treatment after the initial line were excluded (234 patients). The likelihood that these excluded patients actually had advanced CCA was assumed to be low.

This study underscores the limitations of current treatment options for patients after failure of initial gemcitabine- or 5-FU-based therapy and the economic burden associated with previously-treated advanced CCA. There is a need for safe and effective treatments that will improve outcomes and minimize the burden of illness associated with CCA.

\section{Acknowledgments}

Referenced with permission from The NCCN Clinical Practice Guidelines in Oncology (NCCN Guidelines ${ }^{\circledR}$ ) for Hepatobiliary Cancers Version 5.2020. (C) National
Comprehensive Cancer Network, Inc. 2020. All rights reserved. Accessed August 4, 2020. To view the most recent and complete version of the guideline, go online to NCCN. org. NCCN makes no warranties of any kind whatsoever regarding their content, use or application and disclaims any responsibility for their application or use in any way. Editorial support was provided by Gloria DeWalt from Analysis Group, Inc.

Funding: This work was supported by Agios Pharmaceuticals, Inc., Cambridge, MA.

\section{Footnote}

Reporting Checklist: The authors have completed the STROBE reporting checklist. Available at: http://dx.doi. org/10.21037/jgo-20-544

Conflicts of Interest: All authors have completed the ICMJE uniform disclosure form (available at: http://dx.doi. org/10.21037/jgo-20-544). EF and DG are employed by Analysis Group, Inc., which provided consulting services to Agios Pharmaceuticals, Inc. DM was employed by Analysis Group, Inc. during the conduct of the study, which provided consulting services to Agios Pharmaceuticals, Inc. CXC, NW, and ANB are employed by and stockholders in Agios Pharmaceuticals, Inc. MLP reports grants from NIH and personal fees from Agios Pharmaceuticals, Inc. during the conduct of the study. MLP received personal fees from Bayer and Exelixis, travel support from Halozyme, AstraZeneca and Exelixis, and institutional research support from Bayer, Taiho, AstraZeneca, BeiGene, Berg, and Merck, all outside the submitted work.

Ethical Statement: The authors are accountable for all aspects of the work in ensuring that questions related to the accuracy or integrity of any part of the work are appropriately investigated and resolved. The study was conducted in accordance with the Declaration of Helsinki (as revised in 2013). The de-identified data did not require approval from an ethics board.

Open Access Statement: This is an Open Access article distributed in accordance with the Creative Commons Attribution-NonCommercial-NoDerivs 4.0 International License (CC BY-NC-ND 4.0), which permits the noncommercial replication and distribution of the article with the strict proviso that no changes or edits are made and the original work is properly cited (including links to both the 
formal publication through the relevant DOI and the license). See: https://creativecommons.org/licenses/by-nc-nd/4.0/.

\section{References}

1. Razumilava N, Gores GJ. Cholangiocarcinoma. Lancet 2014;383:2168-79.

2. Krasinskas AM. Cholangiocarcinoma. Surg Pathol Clin 2018;11:403-29.

3. Ghouri YA, Mian I, Blechacz B. Cancer review: cholangiocarcinoma. J Carcinog 2015;14:1.

4. Patel N, Benipal B. Incidence of cholangiocarcinoma in the USA from 2001 to 2015: a US cancer statistics analysis of 50 states. Cureus 2019;11:e3962.

5. Saha SK, Zhu AX, Fuchs CS, et al. Forty-year trends in cholangiocarcinoma incidence in the U.S.: intrahepatic disease on the rise. Oncologist 2016;21:594-9.

6. Castro FA, Koshiol J, Hsing AW, et al. Biliary tract cancer incidence in the United States-Demographic and temporal variations by anatomic site. Int J Cancer 2013;133:1664-71.

7. Yao KJ, Jabbour S, Parekh N, et al. Increasing mortality in the United States from cholangiocarcinoma: an analysis of the National Center for Health Statistics Database. BMC Gastroenterol 2016;16:117.

8. American Cancer Society. Key statistics for bile duct cancer. Available online: https://www.cancer.org/cancer/bile-ductcancer/about/key-statistics.html. Accessed May 20, 2020.

9. Patel T. Cholangiocarcinoma--controversies and challenges. Nat Rev Gastroenterol Hepatol 2011;8:189-200.

10. Cardinale V, Bragazzi MC, Carpino G, et al. Cholangiocarcinoma: increasing burden of classifications. Hepatobiliary Surg Nutr 2013;2:272-80.

11. American Cancer Society. Survival rates for bile duct cancer. Available online: https://www.cancer.org/cancer/bile-ductcancer/detection-diagnosis-staging/survival-by-stage.html. Accessed May 20, 2020.

12. Abou-Alfa GK, Macarulla T, Javle MM, et al. Ivosidenib in IDH1-mutant, chemotherapy-refractory cholangiocarcinoma (ClarIDHy): a multicentre, randomised, double-blind, placebo-controlled, phase 3 study. Lancet Oncol 2020;21:796-807.

13. Abou-Alfa GK, Sahai V, Hollebecque A, et al. Pemigatinib for previously treated, locally advanced or metastatic cholangiocarcinoma: a multicentre, open-label, phase 2 study. Lancet Oncol 2020;21:671-84.

14. Rizvi S, Khan SA, Hallemeier CL, et al. Cholangiocarcinoma - evolving concepts and therapeutic strategies. Nat Rev Clin Oncol 2018;15:95-111.
15. Khan SA, Davidson BR, Goldin RD, et al. Guidelines for the diagnosis and treatment of cholangiocarcinoma: an update. Gut 2012;61:1657-69.

16. Valle J, Wasan H, Palmer DH, et al. Cisplatin plus gemcitabine versus gemcitabine for biliary tract cancer. N Engl J Med 2010;362:1273-81.

17. National Comprehensive Cancer Network, Hepatobiliary Cancers Version 5.2020.

18. Lamarca A, Palmer DH, Wasan HS, et al. ABC-06 I A randomised phase III, multi-centre, open-label study of active symptom control (ASC) alone or ASC with oxaliplatin / 5-FU chemotherapy (ASC+mFOLFOX) for patients (pts) with locally advanced / metastatic biliary tract cancers $(\mathrm{ABC})$ previously-treated with cisplatin/gemcitabine (CisGem) chemotherapy. J Clin Oncol 2019;37:4003.

19. PEMAZYRE ${ }^{\mathrm{TM}}$ (pemigatinib) Highlights of prescribing information. Available online: https://www.pemazyre.com/ pdf/prescribing-information.pdf. Accessed June 24, 2020.

20. Wadhwa V, Jobanputra Y, Thota PN, et al. Healthcare utilization and costs associated with cholangiocarcinoma. Gastroenterol Rep (Oxf) 2017;5:213-8.

21. Lee R. National trends in cholangiocarcinoma in the United States (US): 2000-2014. J Clin Oncol 2017;35:e13056.

22. U.S. Bureau of Labor Statistics. Consumer Price Index. Available online: https://www.bls.gov/cpi/. Accessed May 20, 2020.

23. Ransome E, Tong L, Espinosa J, et al. Trends in surgery and disparities in receipt of surgery for intrahepatic cholangiocarcinoma in the US: 2005-2014. J Gastrointest Oncol 2019;10:339-47.

24. Chakunta HR, Sunderkrishnan R, Kaplan MA, et al. Cholangiocarcinoma: treatment with sorafenib extended life expectancy to greater than four years. J Gastrointest Oncol 2013;4:E30-2.

25. Rogers JE, Law L, Nguyen VD, et al. Second-line systemic treatment for advanced cholangiocarcinoma. J Gastrointest Oncol 2014;5:408-13.

26. Graham RP, Barr Fritcher EG, Pestova E, et al. Fibroblast growth factor receptor 2 translocations in intrahepatic cholangiocarcinoma. Hum Pathol 2014;45:1630-8.

27. Ross JS, Wang K, Gay L, et al. New routes to targeted therapy of intrahepatic cholangiocarcinomas revealed by next-generation sequencing. Oncologist 2014;19:235-42.

28. Farshidfar F, Zheng S, Gingras MC, et al. Integrative genomic analysis of cholangiocarcinoma identifies distinct IDH-mutant molecular profiles. Cell Rep 2017;19:2878-80.

29. Boscoe AN, Rolland C, Kelley RK. Frequency and prognostic significance of isocitrate dehydrogenase 1 
mutations in cholangiocarcinoma: a systematic literature review. J Gastrointest Oncol 2019;10:751-65.

30. Ting J, Tien Ho P, Xiang P, et al. Cost-effectiveness and value of information of erlotinib, afatinib, and cisplatinpemetrexed for first-line treatment of advanced EGFR mutation-positive non-small-cell lung cancer in the United
States. Value Health 2015;18:774-82.

31. Larsen FO, Markussen A, Diness LV, et al. Efficacy and safety of capecitabine, irinotecan, gemcitabine, and bevacizumab as second-line treatment in advanced biliary tract cancer: a phase II study. Oncology 2018;94:19-24.
Cite this article as: Chamberlain CX, Faust E, Goldschmidt D, Webster N, Boscoe AN, Macaulay D, Peters ML. Burden of illness for patients with cholangiocarcinoma in the United States: a retrospective claims analysis. J Gastrointest Oncol 2021;12(2):658-668. doi: 10.21037/jgo-20-544 


\section{CCA Diagnosis}

Patients required to have at least 3 diagnosis codes for CCA on distinct days within one year of the first CCA-related claim observed in the data
Index date

The last administration of the firstline systemic therapy (gemcitabine or 5-FU-based treatment) plus 28 days or the initiation of second-line therapy

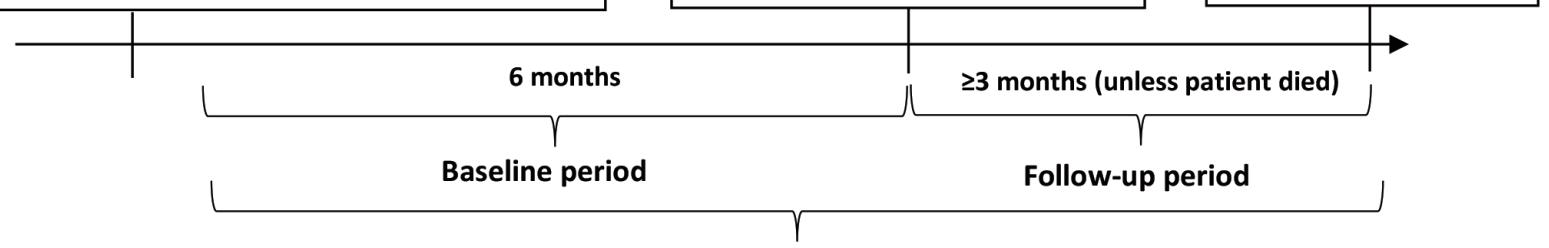

\section{Study period}

Abbreviations: 5-FU = fluorouracil; $\mathrm{CCA}=$ cholangiocarcinoma

Figure S1 Study schematic.

Table S1 CCA-related HRU during the follow-up period stratified by age

$\begin{array}{lr}<65 \text { years } & \geq 65 \text { years } \\ (\mathrm{N}=359) & (\mathrm{N}=939)\end{array}$

\footnotetext{
Number of CCA-related medical services per patient per month ${ }^{1,2}$, mean \pm SD

Inpatient admissions

Inpatient length of stay per admission ${ }^{3}$

ICU admissions

ICU length of stay per admission ${ }^{3}$

Emergency room visits

Home health care/hospice visits

Outpatient visits

Radiology visits

$0.2 \pm 0.5$

$2.8 \pm 6.5$

$0.1 \pm 0.2$

$1.7 \pm 2.8$

$0.0 \pm 0.1$

$0.1 \pm 0.4$

$1.3 \pm 2.6$

$0.3 \pm 0.4$

$0.2 \pm 1.3$

$5.3 \pm 20.7$

$0.1 \pm 1.1$

$5.2 \pm 13.1$

$0.0 \pm 0.1$

$0.1 \pm 0.3$

$0.9 \pm 1.6$

$0.2 \pm 0.4$

Abbreviations: $\mathrm{CCA}$ = cholangiocarcinoma; $\mathrm{HRU}=$ healthcare resource utilization; $\mathrm{ICU}=$ intensive care unit; $\mathrm{SD}=\mathrm{standard}$ deviation Notes:

${ }^{1}$ If any claim within an admission/visit had a diagnosis code for CCA, the entire visit was considered CCA-related.

${ }^{2}$ Per patient per month HRU was calculated based on the average of the number of admission(s) or visit(s) per patient divided by number of months in each patient's follow-up.

${ }^{3}$ Length of stay for inpatient and ICU admissions were calculated only among patients with at least one inpatient or ICU admission, respectively.
} 
Table S2 Monthly CCA-related healthcare costs per patient during the follow-up period stratified by age

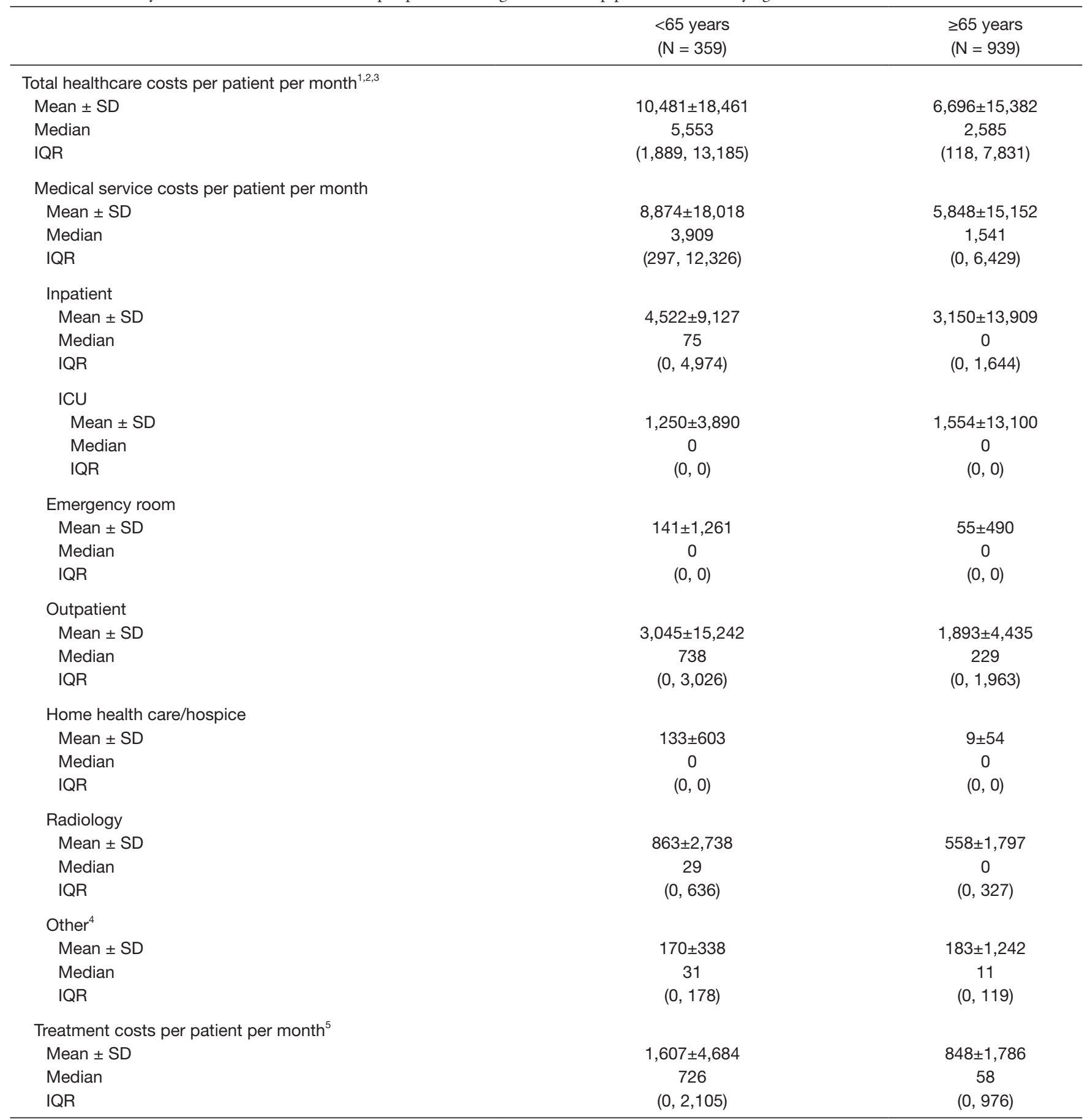

Abbreviations: CCA = cholangiocarcinoma; ICU = intensive care unit; SD = standard deviation; USD = United States dollars Notes:

${ }^{1}$ If any claim within a visit had a diagnosis code for CCA, the entire visit was considered CCA-related.

${ }^{2}$ All costs were inflated to 2019 USD.

${ }^{3}$ Per patient per month costs were calculated based on the average of the costs per patient for each admission or visit type divided by number of months in each patient's follow-up.

4 "Other refers to the following types of services: durable medical equipment, services and supplies, transportation services, laboratory services, and dental services.

${ }^{5}$ CCA-related treatment costs included systemic treatments only. 